\title{
Anatomical Structures Segmentation by Spherical 3D Ray Casting and Gradient Domain Editing
}

\author{
A. Kronman ${ }^{1}$, Leo Joskowicz ${ }^{1}$, and J. Sosna ${ }^{2}$ \\ 1 School of Eng. and Computer Science, The Hebrew Univ. of Jerusalem, Israel \\ 2 Dept. of Radiology, Hadassah Hebrew University Medical Center, Jerusalem, Israel \\ achiak@cs.huji.ac.il
}

\begin{abstract}
Fuzzy boundaries of anatomical structures in medical images make segmentation a challenging task. We present a new segmentation method that addresses the fuzzy boundaries problem. Our method maps the lengths of $3 \mathrm{D}$ rays cast from a seed point to the unit sphere, estimates the fuzzy boundaries location by thresholding the gradient magnitude of the rays lengths, and derives the true boundaries by Laplacian interpolation on the sphere. Its advantages are that it does not require a global shape prior or curvature based constraints, that it has an automatic stopping criteria, and that it is robust to anatomical variability, noise, and parameters values settings. Our experimental evaluation on 23 segmentations of kidneys and on 16 segmentations of abdominal aortic aneurysms (AAA) from CT scans yielded an average volume overlap error of $12.6 \%$ with respect to the ground-truth. These results are comparable to those of other segmentation methods without their underlying assumptions.
\end{abstract}

\section{Introduction}

Patient-specific models of anatomical structures and pathologies generated from volumetric CT/MRI medical images play an increasingly central role in all aspects of patient care, from the initial diagnosis through the planning, delivery, and evaluation of patient treatment. A key task in the generation of these models is the segmentation of anatomical structures and pathologies of interest.

Anatomical structures and pathologies segmentation is a challenging task. Experience shows that each anatomical structure, pathology, and imaging modality has unique characteristics that may lead to significant segmentation errors in a non-negligible number of cases. Correcting faulty segmentations often requires extensive user interaction, trial-and-error parameter tuning, and/or developing custom algorithms, all of which are impractical in a clinical environment.

One of the main reasons that segmentation is challenging is the existence of fuzzy boundaries between the structure of interest and its neighboring structures (Fig. 1a). This occurs when the voxels intensity gradient magnitudes of the target and its neighboring structures boundaries are small, i.e., when their tissue imaging characteristics are similar and when partial volume effect, and/or imaging 


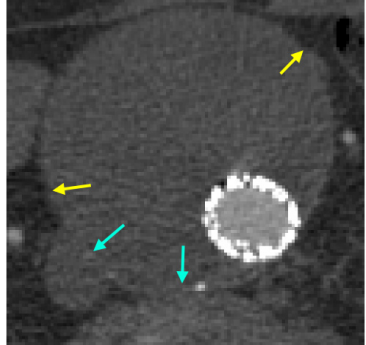

(a)

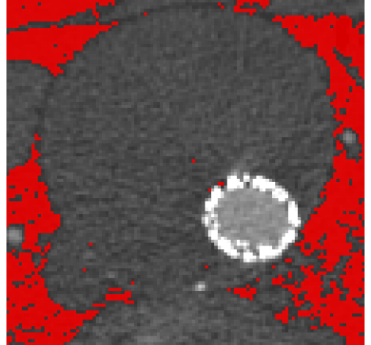

(b)

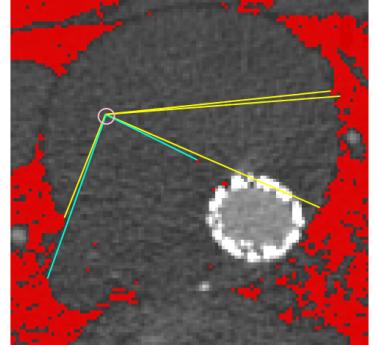

(c)

Fig. 1. Illustration on an AAA axial CT slice: (a) original slice - the arrows show sharp (yellow) and fuzzy (cyan) boundaries; (b) result of conservative intensity-based background (red) thresholding to the image; (c) rays cast from a seed point (purple circle) stop at the true boundary (yellow rays) or at a false boundary (cyan rays). Note that for two neighbor rays, their length difference is large when one ray stops at the true boundary and the other ray does not.

noise blurs the image. As a result, the segmentation volume may expand outside the target structure boundary into neighboring structures, thus producing what is termed as segmentation leaks. Fuzzy boundaries cause segmentation leaks in nearly all segmentation methods. In intensity-based thresholding methods, the thresholding classification rule is based on statistically significant intensity differences between neighboring structures, so leaks will appear in fuzzy boundaries. In adaptive region growing [1] and ray casting methods [2, leaks occur when the intensity based stopping criteria does not apply for the fuzzy boundaries voxels. Intensity-based graph-cut methods may produce leaks because the mincut is unlikely to separate voxels with similar intensities, as this penalizes the global energy function. Energy-based methods, including snakes and level-set active contours, may also produce leaks since they move away the evolving segmentation contour in low-gradient regions because the image term in the energy equation yields a higher overall energy.

Various methods have been proposed to address the fuzzy boundaries problem. The most popular ones incorporate global shape priors 34 and curvature-based constraints [56] into the segmentation. While global shape priors are useful, they have the following limitations: 1) they can not handle pathologies that have no representative shape; 2) they may not converge for far-from-average cases, and; 3) they are difficult to acquire, since they depend on the availability of a delineated atlas. Adding curvature-based constraints into energy-based segmentation methods helps to avoid segmentation leaks when the leak region has a narrow bottleneck shape. However, since the stopping criteria has to be tailored anew for each specific structure, the user often needs to actively stop the segmentation process, which may be inaccurate and inconsistent, and is thus undesirable.

Dodin et al. 2] describe a 2D ray casting method for the segmentation of knee bones in MRI scans. Their rays stopping criteria is based on the Laplacian zero-crossing, so their method will produce segmentation leaks in structures with 
extended fuzzy boundaries caused by adjacent structures with similar intensity values. Moreover, 2D ray casting requires at least one seed point for each scan slice, which may be impractical.

In this paper we present a new seeds-based 3D ray casting segmentation method that handles fuzzy boundaries without shape priors or curvature constraints. Its advantages are that: 1) it does not require a global shape prior; 2) it does not assume a bottleneck-like shape of leaks; 3) it requires a few seeds; 4) it does not rely on the user to actively stop the segmentation; 5) it is robust to anatomical variability, noise and segmentation parameters; and, 6) it is applicable to a variety of anatomical structures. Our experimental study on segmentations of 23 kidneys and 16 abdominal aortic aneurysms (AAA) from CT scans yielded an average volume overlap error of $12.6 \%$ with respect to their ground-truth segmentations. These results are comparable to other segmentation methods which require shape priors or curvature penalties, but without parameters adjustment and underlying assumptions.

\section{Method}

The algorithm inputs are one or more seeds located inside the target structure - the seeds are either provided by the user or generated automatically. For each seed point, a segmentation is generated in three steps: 1) initial segmentation by spherical 3D ray casting; 2) identification of correct/incorrect segmentation regions by spherical discontinuation computation; and 3) correction of the faulty boundaries by Laplacian interpolation on the unit sphere. The segmentation results for each seed are combined to produce the final segmentation.

\subsection{Initial Segmentation by Spherical 3D Ray Casting}

Let $I=\left\{I_{i}\right\}$ be a volumetric image consisting of $n$ voxels $I_{i}$. Let $T=\left\{T_{i}\right\}$ be a target structure of interest in $I$, and let $S=\left\{S_{i}\right\}$ be the unknown surface of $T$. We divide the image $I$ into two disjoint subsets based on a surface thresholding criteria: the sharp voxels set $C=\left\{C_{i}\right\}$, and the fuzzy voxels set $F=\left\{F_{i}\right\}$ (Fig. 1b). The thresholding can be based on gray-level values, gradient magnitudes, the output of an edge detector, or any other suitable criteria.

The inputs to the algorithm are $k$ predefined seed points $p_{1}, \ldots, p_{k}$ located inside the target structure (Fig. 2a). For each seed point, the initial segmentation is computed as follows. For each angle $0 \leq \theta_{i} \leq 2 \pi$ and $0 \leq \phi_{i} \leq \pi$, a 3D ray emanating from the seed in the direction defined by $\theta_{i}$ and $\phi_{i}$ is cast until it hits a voxel in $C$. The resulting segmentation is the total volume covered by the rays. Note that this initial segmentation includes both parts of the true boundary $S$ and also faulty boundary segments resulting from fuzzy boundaries and/or noise (Figs. 2b,3e,3g). We choose this segmentation method because it enables us to automatically estimate and correct the segmentation errors in the next steps. 
(a)

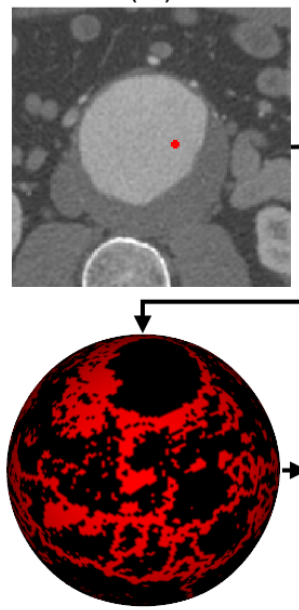

(e) (b)

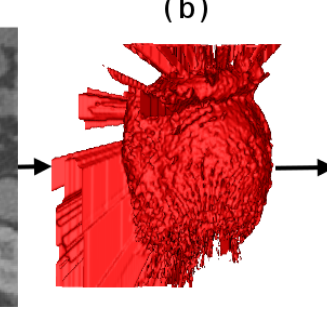

(c)

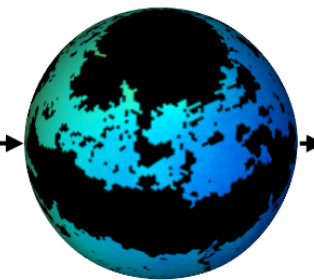

( f)

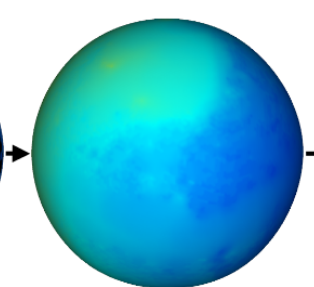

(g) (d)

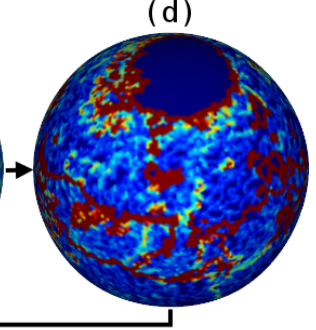

Fig. 2. Illustration of the algorithm: (a) AAA CT axial slice with a seed point (red dot); (b) initial mesh generated by spherical 3D ray casting segmentation; (c) rays length mapping to the sphere - warm (red) colors indicate long rays; (d) rays length gradient magnitude; (e) gradient magnitude thresholding - nodes with large gradient values are red; (f) rays length on the largest connected component; (g) estimation of the unknown boundaries by Laplacian interpolation; (h) final corrected segmentation

\subsection{Errors Estimation by Spherical Discontinuation Computation}

Next, we classify the cast rays into those that stopped at the true boundary $S$ and those that did not. The estimation is based on the following observation. For each orientation $\left(\theta_{i}, \phi_{i}\right)$, the rays will stop at the true boundary $S$ with the exception of orientations where there exist a voxel that belongs to $C$ in the interior of $T$ or for directions where the boundary $S$ is fuzzy, i.e. when it does not belong to $C$. In the first case, the rays will stop before the true boundary, while in the second case the rays will stop after the true boundary (Fig. 1c). As a result, the rays length difference for two voxels in sequential orientations will be relatively large when one of the voxels belongs to the true boundary $S$ and the other does not. In contrast, this difference will be much smaller when both voxels belong to the true boundary.

We formalize this observation to identify the incorrect boundaries of the initial segmentation as follows. Let $f:[0,2 \pi] \times[0, \pi] \rightarrow \Re^{+}$be a scalar function on the unit sphere such that $f(i, j)=l_{i j}$ where $l_{i j}$ is the length of the ray cast in the direction $\left(\theta_{i}, \phi_{j}\right)$.

Following [7], we sample $f$ uniformly by constructing a triangulated mesh approximation of the unit sphere by icosahedron subdivision [8] centered around the origin. The result is a geodesic sphere whose nodes are equidistant from each other and have no interpolation singularities around the sphere poles. We associate to 
each node its matching $f$ values, $f\left(S p_{i}\right)=l_{j k}$, where $S p_{i}$ is a node in the generated mesh $S p$ and $l_{j k}$ is the length of the ray cast in the direction defined by $S p_{i}$ spherical coordinates $\left(\theta_{j}, \phi_{k}\right)$ relative to the seed point (Fig. 2c).

Next, we find the sharp changes in $f$ by computing for each mesh node $S p_{i}$ its gradient magnitude:

$$
\left|\nabla f\left(S p_{i}\right)\right|=\sqrt{\sum_{S p_{j} \in N_{i}}\left(f\left(S p_{i}\right)-f\left(S p_{j}\right)\right)^{2}}
$$

where $N_{i}$ are the neighboring nodes of $S p_{i}$ (Fig. 2d). Based on the previous observation, we compute the set of nodes $B \subseteq S p$ that match to the interface between the correct and faulty initial segmentation by thresholding:

$$
B=\left\{S p_{i}:\left|\nabla f\left(S p_{i}\right)\right|>t\right\}
$$

where $t>0$ is a preset constant with a fixed value for every image (Fig. 2e).

Finally, we isolate the sphere nodes that correspond to orientations with correct initial segmentation by choosing the largest nodes connected component that are separated by nodes in $B$ (Fig. 2f).

\subsection{Correct Boundaries Detection by Laplacian Spherical Interpolation}

This step detects the true boundaries in the directions where the initial segmentation was incorrect (Fig. $2 \mathrm{~g}$ ). For this we use Laplace interpolation, which minimizes the integrated square of the gradients of $f$, and thus yields the smoothest surface with respect to this criteria. The Laplace interpolation with Dirichlet boundary condition is formulated as:

$$
\nabla^{2} f\left(S p_{i}\right)=0, \quad \text { subject to: } \quad f\left(S p_{j}\right)=f^{*}\left(S p_{j}\right)
$$

for each unknown node $S p_{i}$ on the mesh and for each node $S p_{j}$ on the boundaries of the interpolation domain with a known value $f^{*}\left(S p_{j}\right)$, where $\nabla^{2} f\left(S p_{i}\right)=$ $\sum_{S p_{k} \in N_{i}}\left(f\left(S p_{i}\right)-f\left(S p_{k}\right)\right)$ is the discrete graph Laplacian operator [9], and $N_{i}$ are the neighbors of node $S p_{i}$ on the sphere mesh $S p$. The unknown values $f\left(S p_{i}\right)$ are then computed by solving the resulting sparse system of linear equations.

We generate the final segmentation with the following classification rule to each image voxel $I_{l}$ at orientations $\left(\theta_{i}, \phi_{j}\right)$ relative to seed point $p_{k}$ :

$$
L_{k}\left(I_{l}\right)= \begin{cases}1 & \text { if } d\left(p_{k}, I_{l}\right) \leq \hat{f_{i j}} \\ 0 & \text { if } d\left(p_{k}, I_{l}\right)>\hat{f_{i j}}\end{cases}
$$

where $L_{k}\left(I_{l}\right)$ is the label for voxel $I_{l}$ for seed $p_{k}, d\left(p_{k}, I_{l}\right)$ is the Euclidean distance between the voxel $I_{l}$ and the seed point $p_{k}$, and $\hat{f_{i j}}$ is the distance interpolation solution for orientations $\left(\theta_{i}, \phi_{j}\right)$. Figs. $2 \mathrm{~h}, 3 \mathrm{f}, 3 \mathrm{~h}$ show examples of the results.

The key advantage of this new estimation by interpolation method is that any remaining false positive estimation of a leak region caused by choosing to lower 
the thresholding parameter $t$ in (2) will be set to its original, correctly estimated value.

Note that the segmentation by ray-casting just described applies to voxels that are visible from the seed point $p_{k}$, i.e. to a convex or star-shaped objects. We directly extend the method to general shapes by taking the union of the segmentations $L_{i}$ obtained for each seed $p_{i}$ :

$$
L_{\text {final }}\left(I_{l}\right)=\bigvee_{i=1}^{k} L_{i}\left(I_{l}\right)
$$

The number of seeds required for the structure segmentation depends on the number of its star-shaped components. For the structures of interest, a few seed points are sufficient to segment the structure appropriately.

\section{Experimental Results}

To quantify the scope, accuracy, and robustness of our method, we designed and conducted the following experimental study. We retrospectively selected 28 CT scans of two different structures, kidneys (organ) and aortic arch aneurysms (AAA - vascular pathology). These structures are different in shape, intensity homogeneity, and surrounding structures. For the kidney study, we included 12 clinical CT datasets (2 kidneys per scan) of size $512 \times 512 \times 350-500$ voxels, each of size $0.5-1.0 \times 0.5-1.0 \times 1.0-1.5 \mathrm{~mm}^{3}$, with and without contrast agent, acquired with a Brilliance 64-row CT scanner (Phillips Healthcare, Cleveland, OH). Ground-truth segmentations of the kidneys were obtained from three manual segmentations of experts with STAPLE 10. For the AAA, we included 16 clinical CT scans of size $512 \times 512 \times 500$ voxels, each of size $0.7-1.2 \times 0.7-1.2 \times 0.7-1.2 \mathrm{~mm}^{3}$ from the same scanner. Imaging streaking artifacts caused by implanted stents were present in several scans (Fig. 3c). An expert radiologist manually generated the ground-truth segmentations.

The method internal parameters values where fixed for all scans to constant values. The background thresholding level was set from the intensity levels, where each voxel with gray-value $<0 \mathrm{HU}$ is a surface candidate. The rays lengths gradient thresholding constant was set to $t=3 \mathrm{~mm}$. We found empirically that these values minimize the false negative leaks detection rate. Note that with these parameters setup up to $50 \%$ of the target surface was considered as "fuzzy". The number of the icosahedron subdivisions was set to 6 . The program runs on a $64-$ bit quad-core $2.80 \mathrm{GHz}$ processors and $6 \mathrm{~GB}$ memory PC. The seeds were placed interactively by the user: after each seed placement the volume that is seen by the seed was marked on the image until the entire target volume was covered. The mean number of seeds required for the segmentation was 3 ( $\operatorname{std}=0.69$ ) and $1.33(\mathrm{std}=0.5)$ for the kidney and the AAA. The mean running time was 35.7 $(\operatorname{std}=2.7)$ secs and 41.1 ( $\operatorname{std}=10.7)$ secs for the kidney and the AAA.

To quantify the accuracy of our method, we used both volumetric and surface based metrics. For the kidney, the mean absolute volume difference was $6.02 \%$ 


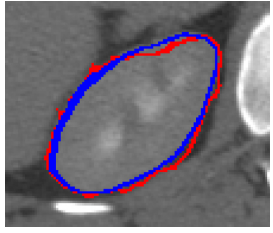

(a)

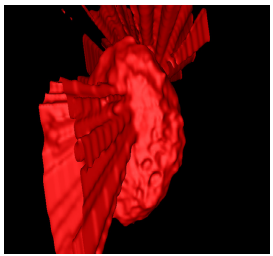

(e)

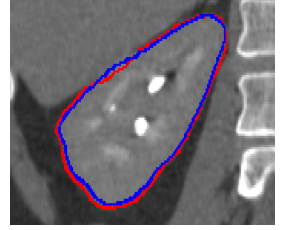

(b)

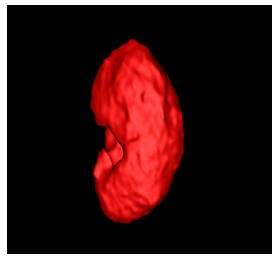

(f)

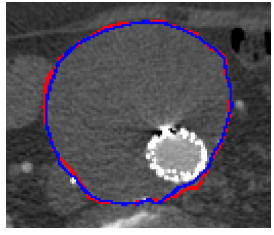

(c)

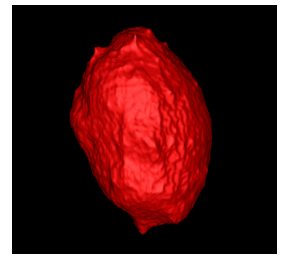

(g)

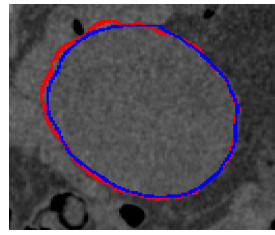

(d)

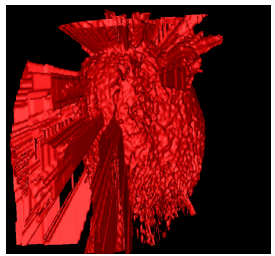

(h)

Fig. 3. (a-d): representative results of our segmentation method (red) and manual ground truth (blue): (a) kidney axial CT slice; (b) kidney coronal slice; (c) AAA axial slice; (d) AAA coronal slice; (e-h): 3D meshes of kidney (e-f) and AAA (g-h): (e,g) $3 \mathrm{D}$ meshes generated by spherical 3D ray casting; (f,h) final meshes after applying our leaks correction method

$(\operatorname{std}=4.07 \%)$, the mean volumetric overlap error was $12.65 \%(\operatorname{std}=2.46 \%)$ and the average symmetric surface distance was $1.12(\mathrm{std}=0.4) \mathrm{mm}$. For the AAA, the absolute volume difference was $7.99 \%$ ( $\mathrm{std}=8 \%$ ), the mean volumetric overlap error was $12.63 \%(\mathrm{std}=4.35 \%)$, and the average symmetric surface distance was $1.29(\operatorname{std}=0.3) \mathrm{mm}$. Fig. 3 shows a representative example. These results are comparable to previously reported methods 11 12 13 14 without their underlying assumptions and with a significant improvement in the running time.

\section{Conclusion}

We have developed a new method for the segmentation of anatomical structures in medical images. Our method is based on 3D spherical ray casting and segmentation leaks correction by editing the gradients of the cast rays lengths mapped to the unit sphere. Our experimental study on segmentations of 23 kidneys and 16 abdominal aortic aneurysms from CT scans yielded a mean volume overlap error of $12.6 \%$ with respect to the ground-truth. This was deemed clinically acceptable for diagnosis and surgery planning by the co-author expert radiologist.

The advantages of our method are that it does not require a global shape prior, that it does not assume bottleneck-like shape of the leak, that it does not rely on the user to actively stop the segmentation, that it is robust to anatomical variability, noise and segmentation parameters, and that it is applicable to a variety of anatomical structures. Its limitation is that it requires a predefined seed point for each star-shaped component of the target structure. However, for 
structures such as the kidney and the AAA, only a few (1-4) seeds are needed in practice. Ongoing and future work includes the evaluation of the method on additional anatomical structures.

\section{References}

1. Pohle, R., Toennies, K.: Segmentation of medical images using adaptive region growing. In: Proc. SPIE Medical Imaging, vol. 4322, pp. 1337-1346 (2001)

2. Dodin, P., Martel-Pelletier, J., Pelletier, J., Abram, F.: A fully automated human knee $3 \mathrm{~d}$ mri bone segmentation using the ray casting technique. In: Medical and Biological Engineering and Computing, pp. 1-12 (2011)

3. Freedman, D., Zhang, T.: Interactive graph cut based segmentation with shape priors. In: IEEE Computer Society Conference on Computer Vision and Pattern Recognition, CVPR 2005, vol. 1, pp. 755-762 (2005)

4. Tsai, A., Yezzi Jr., A., Wells, W., Tempany, C., Tucker, D., Fan, A., Grimson, W., Willsky, A.: A shape-based approach to the segmentation of medical imagery using level sets. IEEE Transactions on Medical Imaging 22(2), 137-154 (2003)

5. Djabelkhir, F., Khamadja, M., Odet, C.: Level set constrained segmentation using local curvature. In: 5th International Symposium on Image and Signal Processing and Analysis, ISPA, 152-155. IEEE (2007)

6. El-Zehiry, N., Grady, L.: Fast global optimization of curvature. In: 2010 IEEE Conference on Computer Vision and Pattern Recognition (CVPR), pp. 3257-3264 (2010)

7. Styner, M., Oguz, I., Xu, S., Brechbuhler, C., Pantazis, D., Levitt, J.J., Shenton, M.E., Gerig, G.: Framework for the statistical shape analysis of brain structures using spharm-pdm. Insight J. (1071), 242-250 (2006)

8. Loop, C.: Smooth subdivision surfaces based on triangles (1987)

9. Reuter, M., Biasotti, S., Giorgi, D., Patanč, G., Spagnuolo, M.: Discrete laplacebeltrami operators for shape analysis and segmentation. Computers \& Graphics 33(3), 381-390 (2009)

10. Warfield, S., Zou, K., Wells, W.: Simultaneous truth and performance level estimation (staple): an algorithm for the validation of image segmentation. IEEE Transactions on Medical Imaging 23(7), 903-921 (2004)

11. Freiman, M., Kronman, A., Esses, S., Joskowicz, L., Sosna, J.: Non-parametric Iterative Model Constraint Graph min-cut for Automatic Kidney Segmentation. In: Jiang, T., Navab, N., Pluim, J.P.W., Viergever, M.A. (eds.) MICCAI 2010, Part III. LNCS, vol. 6363, pp. 73-80. Springer, Heidelberg (2010)

12. Rao, M., Stough, J., Chi, Y., Muller, K., Tracton, G., Pizer, S., Chaney, E.: Comparison of human and automatic segmentations of kidneys from ct images. Int. J. Radiat. Oncol. Biol. Phys. 61(3), 954-960 (2005)

13. Lin, D., Lei, C., Hung, S.: Computer-aided kidney segmentation on abdominal ct images. IEEE Transactions on Information Technology in Biomedicine 10(1), 59-65 (2006)

14. Freiman, M., Esses, S.J., Joskowicz, L., Sosna, J.: An iterative model-constraint graph-cut algorithm for abdominal aortic aneurysm thrombus segmentation. In: Proc. of the 2010 IEEE Int. Symp. on Biomedical Imaging, ISBI 2010 (2010) 